\title{
ON THE EXISTENCE OF SOLUTIONS FOR VOLTERRA INTEGRAL INCLUSIONS IN BANACH SPACES ${ }^{1}$
}

\author{
EVGENIOS P. AVGERINOS \\ University of the Aegean \\ Department of Mathematics \\ Karlovassi 83200, Samos, GREECE
}

\begin{abstract}
In this paper we examine a class of nonlinear integral inclusions defined in a separable Banach space. For this class of inclusions of Volterra type we establish two existence results, one for inclusions with a convex-valued orientor field and the other for inclusions with nonconvexvalued orientor field. We present conditions guaranteeing that the multivalued map that represents the right-hand side of the inclusion is $\alpha$ condensing using for the proof of our results a known fixed point theorem for $\alpha$-condensing maps.
\end{abstract}

Key words: Volterra integral inclusions, Aumann selection theorem, radial retraction, $\alpha$-condensing map.

AMS (MOS) subject classifications: $\quad 35 \mathrm{R} 15,34 \mathrm{G} 20,34 \mathrm{~A} 60$.

\section{INTRODUCTION-PRELIMINARIES}

In this paper we examine a class of nonlinear integral inclusions defined in a separable Banach space and we establish two existence results. One for inclusions with a convex-valued orientor field and the other for inclusions with a nonconvex valued orientor field. Our work extends existence results of Ragimkhanov [11] and Lyapin [7] and the infinite dimensional results of Chuong [3] and Papageorgiou [10], where the hypotheses on the orientor field $F(t, x)$ are too restrictive (see theorem 3.1 of Chuong and theorems 3.1-3.3 of Papageorgiou).

Let $(\Omega, \Sigma)$ be a measurable space and $X$ a separable Banach space. Throughout this work we will be using the following notations:

$$
P_{f(c)}=\{A \subseteq X: \text { nonempty, closed (convex) }\}
$$

\footnotetext{
${ }^{1}$ Received: August, 1991. Revised: April 1993.
} 
and

$$
P_{(w) k(c)}(X)=\{A \subseteq X: \text { nonempty, }(w) \text { compact, }(\text { convex })\} .
$$

A multifunction $F: \Omega \rightarrow P_{f}(X)$ is said to be measurable (see Wagner [13]), if for every $x \in X, \omega \rightarrow d(x, F(\omega))=\inf \{\|x-z\|: z \in F(\omega)\}$ is measurable. When there is a $\sigma$-field measure $\mu(\cdot)$ on $(\Omega, \Sigma)$ and $\Sigma$ is $\mu$-complete, then the above definition of measurability is equivalent to saying that $G r F=\{(\omega, x) \in \Omega \times X: x \in F(\omega)\} \in \Sigma \times B(X)$, with $B(X)$ being the Borel $\sigma$-field of $X$ (graph measurability).

By $S_{F}$ we will denote the set of measurable selectors of $F(\cdot)$ while by $S_{F}^{p}$ $(1 \leq p \leq \infty)$ the set of measurable selectors of $F(\cdot)$ that belong in the LebesgueBochner space $L^{p}(X)$, i.e. $S_{F}^{p}=\left\{f \in L^{p}(X): f(\omega) \in F(\omega) \mu\right.$-a.e. $\}$. This set may be empty. It is nonempty if and only if $\omega \rightarrow \inf \{\|z\|: z \in F(\omega)\} \in L_{+}^{p}$.

In particular this is the case if $\omega \rightarrow|F(\omega)|=\sup \{\|z\|: z \in F(\omega)\} \in L_{+}^{p}$ in which case we say that $F(\cdot)$ is $L^{p}$-integrably bounded.

If $Y, Z$ are Hausdorff topological spaces and $G: Y \rightarrow 2^{Z} \backslash\{\emptyset\}$ then we say that $G(\cdot)$ is lower semicontinuous (l.s.c.), if for all $U \subset Z$ open, the set $G^{-}(U)=\{y \in Y: G(y) \cap U \neq \emptyset\}$ is open in $Y$.

If furthermore $Y, Z$ are metric spaces, then the above definition is equivalent to saying that for all $y_{n} \rightarrow y$ we have $G(y) \subseteq \underline{\lim } G\left(y_{n}\right)=\left\{z \in Z: z=\lim z_{n}, z_{n} \in G\left(y_{n}\right)\right\}$.

Also the multifunction $F: Y \rightarrow 2^{Z} \backslash\{\emptyset\}$ is said to be upper semicontinuous (u.s.c.) if and only if for every $W \subseteq Z$ open, the set $F^{+}(W)=\{y \in Y: F(y) \subset W\}$ is open in $Y$.

Finally we say that a multifunction $G: Y \rightarrow 2^{Z} \backslash\{\emptyset\}$ is closed if and only if the set $G r G=\{(y, z) \in z \in G(y)\}$ is closed in $Y \times Z$.

\section{EXISTENCE THEOREMS}

Let $T=[0, b], b>0$ and let $X$ be a separable Banach space. The integral inclusion of Volterra type which we will be studying is the following:

$$
x(t) \in p(t)+\int_{0}^{t} K(t, s) F(s, x(s)) d s, t \in T
$$


where $p(\cdot) \in C(T, X)$.

By a solution of $(*)$ we understand a function $x(\cdot) \in C(T, X)$ such that

$$
x(t) \in p(t)+\int_{0}^{t} K(t, s) f(s) d s, t \in T, \text { with } f \in S_{F(\cdot, x(\cdot))}^{1} .
$$

First we prove an existence result for the case where the orientor field $F(t, x)$ is convex valued. For that purpose we will need the following hypotheses on the data of $(*)$.

$H(F): F: T \times X \rightarrow P_{w k c}(X)$ is a multifunction such that:

(1) $t \rightarrow F(t, x)$ is measurable,

(2) $x \rightarrow F(t, x)$ is u.s.c. from $X$ into $X_{w}$,

(3) $|F(t, x)| \leq a(t)+b(t)\|x\|$ a.e. with $a(\cdot), b(\cdot) \in L_{+}^{1}$,

(4) for any $\epsilon>0$ and $V \subseteq X$ bounded, there exists $I_{\epsilon} \subseteq T$ open such that $\mu\left(I_{\epsilon}\right) \leq \epsilon$ and $\alpha(F(J \times V)) \leq \sup _{t \in J} \eta(t) \alpha(V)$ for any $J \subseteq T \backslash I_{\epsilon}$ closed and with $\eta(\cdot) \in L_{+}^{1}$.

Remark: We can replace the sublinear growth condition $H(F)(3)$ by a hypothesis of the form "for every $B \subseteq X$ bounded there exists $a_{B}(\cdot) \in L_{+}^{1}$ s.t. $\sup _{x \in B}|F(t, x)| \leq a_{B}(t)$ ". In this case though the existence result is only local.

$H(K): K: T \times T \rightarrow \mathcal{L}(X)$ is continuous (we can have $K$ defined only on $\Delta$ and set $K(t, s)=K(t, t), t \leq s)$.

Now we are ready for our first result:

Theorem 1: If hypotheses $H(F)$ and $H(K)$ hold and $M\|\eta\|_{1} \leq 1$ where

$$
\begin{gathered}
\|K(t, s)\|_{\mathcal{L}} \leq M, \\
\underline{\text { then }}(*) \text { admits a solution. }
\end{gathered}
$$

Proof: First we will establish an a priori bound for the solutions of $(*)$. So let $x(\cdot) \in C(T, X)$ be such a solution. We have:

$$
\|x(t)\| \leq\|p\|_{\infty}+\int_{0}^{t} M|F(s, x(s))| d s
$$

for all $t \in T$ and with $\|K(t, s)\|_{\mathcal{L}} \leq M$ for all $(t, s) \in \Delta$ (see hypothesis $H(K)$ ). 
Using hypothesis $H(F)(3)$, we get:

$$
\|x(t)\| \leq\|p\|_{\infty}+\int_{0}^{t}(M a(s)+M b(s)\|x(s)\|) d s, \quad t \in T .
$$

Invoking Gronwall's inequality, we get $M_{1}>0$ s.t.

$$
\|x(t)\| \leq M_{1}
$$

for all $t \in T$ and all solutions $x(\cdot) \in C(T, X)$ of $(*)$.

Let $\widehat{F}(t, x)=F\left(t, p_{M_{1}}(x)\right)$, with $p_{M_{1}}(\cdot)$ being the $M_{1}$-radial retraction. We will consider the integral inclusion $(*)$ with the orientor field $F(t, x)$ replaced by $\widehat{F}(t, x)$. Note that because of hypothesis $H(F)(1)$ the multifunction $t \rightarrow \widehat{F}(t, x)$ is also measurable. Also recalling that $p_{M_{1}}(\cdot)$ is Lipschitz continuous and using hypothesis $H(F)(2)$, we get from theorem 7.3.11 (ii $)$, p. 87 of Klein-Thompson [5], that $x \rightarrow \widehat{F}(t, x)$ is u.s.c. from $X$ into $X_{w}$. Furthermore $|\widehat{F}(t, x)| \leq a(t)+b(t) M_{1}=\phi(t)$ a.e. with $\phi(\cdot) \in L_{+}^{1}$.

Finally in hypothesis $H(F)(4)$ we have

$$
\alpha(\widehat{F}(J \times V))=\alpha\left(F\left(J \times p_{M_{1}}(V)\right) \leq \sup _{t \in J} \eta(t) \alpha\left(p_{M_{1}}(V)\right) .\right.
$$

But note that $p_{M_{1}}(V) \subseteq \overline{c o n v}[\{0\} \cup V] \Rightarrow \alpha\left(p_{M_{1}}(V)\right)=\alpha[\{0\} \cup V] \leq \alpha(V)$. So we have $\alpha(\widehat{F}(J \times V)) \leq \sup _{t \in J} \eta(t) \alpha(V)$ and so we have checked that $\widehat{F}(t, x)$ satisfies hypothesis $H(F)(4)$.

Set

$$
H=\left\{y \in C(T, X): y(t)=p(t)+\int_{0}^{t} K(t, s) g(s) d s, \quad t \in T,\|g(t)\| \leq \phi(t) \text { a.e. }\right\}
$$

Next let $R: H \rightarrow 2^{H}$ be defined by

$$
R(x)=\left\{y \in C(T, X): y(t)=p(t)+\int_{0}^{t} K(t, s) f(s) d s, t \in T, f \in S_{\hat{F}(\cdot, x(\cdot))}^{1}\right\} .
$$

First we will show that $R(\cdot)$ has nonempty values. Let $\left\{s_{n}\right\}_{n \geq 1}$ be simple functions such that $s_{n}(t) \stackrel{s}{\rightarrow} x(t)$ a.e. in $X$.

Then for each $n \geq 1, t \rightarrow \widehat{F}\left(t, s_{n}(t)\right)$ is measurable (since $t \rightarrow \widehat{F}(t, x)$ is measurable). So by Aumann's selection theorem (see Wagner [13], theorem $5.10)$, we get $f_{n}: T \rightarrow X$ measurable such that $f_{n}(t) \in \widehat{F}\left(t, s_{n}(t)\right)$. Clearly $f_{n} \in L^{1}(X)$. Note that because $\widehat{F}(t, \cdot)$ is u.s.c. from $X$ into $X_{w}$, 


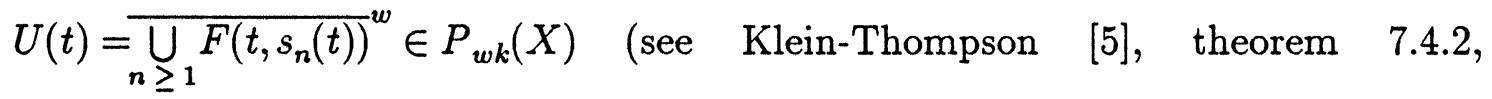
p. 90) and $t \rightarrow U(t)$ is measurable. Hence $t \rightarrow \overline{c o n v} U(t) \equiv U_{c}(t)$ is an integrably bounded, $P_{w k c}(X)$-valued multifunction (Krein-Smulian theorem). So from Papageorgiou [8] (see proposition 3.1), we get that $S_{U_{c}}^{1}$ is $w$-compact in $L^{1}(X)$.

But observe that $\left\{f_{n}\right\}_{n \geq 1} \subseteq S_{U_{c}}^{1}$. So by passing to a subsequence, if necessary, we may assume that $f_{n} \stackrel{w}{\rightarrow} f$ in $L^{1}(X)$. Then from [9] (see theorem 3.1), we get that

$$
\begin{gathered}
f(t) \in \overline{\operatorname{conv}} w-\overline{\lim }\left\{f_{n}(t)\right\}_{n \geq 1} \subseteq \overline{\operatorname{conv}} w-\overline{\lim } \widehat{F}\left(t, s_{n}(t)\right) \\
\subseteq \widehat{F}(t, x(t)) \text { a.e. }
\end{gathered}
$$

the last inclusion following from the upper semicontinuity of $\widehat{F}(t, \cdot)$ from $X$ into $X_{w}$, the fact that $s_{n}(t) \stackrel{s}{\rightarrow} x(t)$ a.e. in $X$ and the fact that $\widehat{F}(\cdot, \cdot)$ is $P_{w k c}(X)$ valued. So $S_{\widehat{F}(\cdot, x(\cdot))}^{1} \neq \emptyset \Rightarrow R(x) \neq \emptyset$ for all $x \in C(T, X)$. Also since $S_{\widehat{F}(\cdot, x(\cdot))}^{1} \in P_{w k c}\left(L^{1}(X)\right)$ (see proposition 3.1 of $[8]$ ), we can easily check that $R(\cdot)$ has closed, convex values in $2^{C(T, X)} \backslash\{\emptyset\}$.

Next we will show that $R(\cdot)$ has a closed graph. To this end let $\left[x_{n}, y_{n}\right] \in G r R$ and assume that $\left[x_{n}, y_{n}\right] \rightarrow[x, y]$ in $C(T, X) \times C(T, X)$. Then by definition for every $n \geq 1$ we have

$$
y_{n}(t)=p(t)+\int_{0}^{t} K(t, s) f_{n}(s) d s, \text { for } t \in T \text { and with } f_{n} \in S_{\widehat{F}\left(\cdot, x_{n}(\cdot)\right)}^{1} .
$$

Note that by the Krein-Smulian theorem (see for example Diestel-Uhl [4], theorem II, p. 51), we have that $\overline{\operatorname{conv}} \bigcup_{n \geq 1} \widehat{F}\left(t, x_{n}(t)\right) \in P_{w k c}(X)$ for all $t \in T$. So from proposition 3.1 of [8] and by passing to a subsequence if necessary, we may assume that $f_{n} \stackrel{w}{\rightarrow} f$ in $L^{1}(X)$. Then as above using theorem 3.1 of [9] and the properties of $\widehat{F}(t, x)$, we get

Also $\int_{0}^{t} K(t, s) f_{n}(s) d s \stackrel{w}{\rightarrow} \int_{0}^{t} K(t, s) f(s) d s$ in $X$. Hence in the limit as $n \rightarrow \infty$

$$
f(t) \in \overline{\operatorname{conv}} w-\overline{\lim }\left\{f_{n}(t)\right\}_{n \geq 1} \subseteq \overline{\operatorname{conv} w} w-\overline{\lim } \widehat{F}\left(t, x_{n}(t)\right) \subseteq \widehat{F}(t, x(t)), \text { a.e. }
$$

$$
y(t)=p(t)+\int_{0}^{t} K(t, s) f(s) d s, t \in T
$$


with $f \in S_{\widehat{F}(\cdot, x(\cdot))}^{1}$. Therefore $[x, y] \in G r R \Rightarrow R(\cdot)$ has a closed graph.

Next by Lusin's theorem, given $\epsilon>0$ there exists $I_{\epsilon}^{1} \subseteq T$ open such that $\lambda\left(I_{\epsilon}^{1}\right)<\epsilon / 2,\left.\eta\right|_{T \backslash I_{\epsilon}^{1}} \in C$ and $\left\|\phi \chi_{I_{\epsilon}}\right\|_{1} \leq \epsilon / 2 M$. Also from hypothesis $H(F)(4)$ (which as we have already checked earlier, is also valid for the orientor field $\widehat{F}(t, x))$, given $V$ a nonempty subset of $H$ we can find $I_{\epsilon}^{2} \subseteq T$ open with $\lambda\left(I_{\epsilon}^{2}\right)<\epsilon / 2$ and

$$
\alpha(F(J \times \widehat{V})) \leq \sup _{s \in J} \eta(s) \alpha(\widehat{V}) \text { and }\left\|\phi \chi_{I_{\epsilon}^{2}}\right\|_{1} \leq \epsilon / 2 M
$$

where $J \subseteq L=T \backslash I_{\epsilon}$ closed, with $I_{\epsilon}=I_{\epsilon}^{1} \cup I_{\epsilon}^{2}$ and $\widehat{V}=\{x(t): x \in V, t \in T\}$.

Note that because of hypothesis $H(K)$ and since by the choice of $\left.L \eta\right|_{L}$ continuous, the map $(s, w) \rightarrow\|K(t, s)\|_{\mathcal{L}} \eta(w)$ is continuous, hence uniformly continuous on $([0, t] \cap L) \times L$. Thus given $\delta>0$ we can find $\theta>0$ s.t.

$$
\left|\|K(t, s)\|_{\mathcal{L}} \eta(w) \alpha(\widehat{V})-\|K(t, \tau)\|_{\mathcal{L}} \eta(z) \alpha(\widehat{V})\right| \leq \delta
$$

for all $s, \tau \in[0, t] \cap L$ with $|s-\tau| \leq \theta$ and all $w, z \in L$ with $|w-z| \leq \theta$.

Let $0=t_{0}<t_{1}<\ldots<t_{n}=b$ be a subdivision of $T$ into $(n+1)$-parts such that $t_{i}-t_{i-1} \leq \theta$ and let $L_{i}=\left[t_{i-1}, t_{i}\right] \backslash I_{\epsilon} \quad i=1,2, \ldots n$.

Also let $v_{i} \in L_{i}$ and $s_{i} \in L_{i} \quad i=1,2, \ldots, n$ be such that

$$
\left\|K\left(t, v_{i}\right)\right\|_{\mathcal{L}}=\sup _{s \in L_{i}}\|K(t, s)\|_{\mathcal{L}}
$$

and $\eta\left(s_{i}\right)=\sup _{s \in L_{i}} \eta(s)$. Their existence is guaranteed by hypothesis $H(K)$ and since $\left.\eta\right|_{L}$ is continuous. Then we have:

$$
\alpha\left[\widehat{F}\left(L_{i} \times \widehat{V}\right)\right] \leq \eta\left(s_{i}\right) \alpha(\widehat{V}) .
$$

Also from the "Mean Value Theorem" for Bochner integrals (see DiestelUhl, [4], corollary 8, p. 48), we have:

$$
\left\{\int_{L_{i}} K(t, s) \widehat{F}(s, x(s)) d s: x \in V\right\} \subseteq \mu\left(L_{i}\right) \overline{\operatorname{conv}}\left[K(t, s) \widehat{F}(s, y): s \in S_{i}, y \in \widehat{V}\right] .
$$

So we have

$$
\left\{\int_{L} K(t, s) \widehat{F}(s, x(s)) d s: x \in V\right\} \subseteq \sum_{i=1}^{n} \mu\left(L_{i}\right) \overline{\operatorname{conv}}\left[K(t, s) \widehat{F}(s, y): s \in S_{i}, \quad y \in \widehat{V}\right] .
$$


Using the subadditivity of the $\alpha(\cdot)$ measure of non-compactness, we get

$$
\begin{gathered}
\alpha\left\{\int_{L} K(t, s) \widehat{F}(s, x(s)) d s: x \in V\right\} \leq \sum_{i=1}^{n} \mu\left(L_{i}\right)\left\|K\left(t, v_{i}\right)\right\|_{\mathcal{L}} \alpha\left[\widehat{F}\left(L_{i}, \widehat{V}\right)\right] \\
\leq \sum_{i=1}^{n} \mu\left(L_{i}\right)\left\|K\left(t, v_{i}\right)\right\|_{\mathcal{L}} \eta\left(s_{i}\right) \alpha(\widehat{V}) .
\end{gathered}
$$

From (1) above we get

$$
\alpha\left\{\int_{L} K(t, s) \widehat{F}(s, x(s)) d s: x \in V\right\} \leq \int_{L}\|K(t, s)\|_{\mathcal{L}} \eta(s) \alpha(\widehat{V}) d \tau+\delta \mu(L) .
$$

Also recall from the initial choice of the sets $I_{\epsilon}^{1}$ and $I_{\epsilon}^{2}$ that

So finally we have:

$$
\int_{I_{\epsilon}}\|K(t, s)\|_{\mathcal{L}} \phi(s) d s \leq \epsilon .
$$

$$
\begin{aligned}
\alpha(R(V)(t)) & \leq \int_{L}\|K(t, s)\|_{\mathcal{L}} \eta(s) \alpha(\widehat{V}) d s+\delta \mu(S)+\epsilon \\
& \leq \int_{0}^{b} M \eta(s) \alpha(\widehat{V}) d s+\delta \mu(S)+\epsilon .
\end{aligned}
$$

Since $\epsilon, \delta>0$ were arbitrary, we get

$$
\alpha(R(V)(t)) \leq \int_{0}^{b} M \eta(s) \alpha(\widehat{V}) d s=\alpha(\widehat{V}) M\|\eta\|_{1}
$$

Since $H$ is bounded and equicontinuous, from Ambrosetti's theorem (see theorem 1.4.2 p. 20 of Lakshmikantham-Leela [6]) we have that

$$
\alpha(\widehat{V}) \leq \widehat{\alpha}(V)
$$

and $\sup _{t \in T} \alpha(R(V)(t))=\widehat{\alpha}(R(V))$. Thus we get

$$
\widehat{\alpha}(R(V)) \leq M\|\eta\|_{1} \widehat{\alpha}(V) .
$$

Since by hypothesis $M\|\eta\|_{1}<1$, we get that $R(\cdot)$ is $\widehat{\alpha}(\cdot)$-condensing. Apply theorem 4.1 of Tarafdar-Vyborny [12], to get $x \in R(x)$. Then $x \in C(T, X)$ solves (*) with the orientor field $\widehat{F}(t, x)$. Using the definition of $\widehat{F}(t, x)$ and same estimation as in the beginning of the proof, we get that

$$
\|x(t)\| \leq M_{1} \Rightarrow \widehat{F}(t, x(t))=F(t, x(t)) \Rightarrow x(\cdot) \in C(T, X) \text { solves }(*) .
$$


We can have a variant of Theorem 1 , where the orientor field is not convex-valued. For this we will need the following hypothesis.

$H(F)^{\prime}: \quad F: T \times X \rightarrow P_{k}(X)$ is a multifunction such that

(1) $(t, x) \rightarrow F(t, x)$ is graph measurable,

(2) $\quad x \rightarrow F(t, x)$ is l.s.c.,

and the hypotheses $H(F)(3)$ and (4) also hold.

Theorem 2: If hypotheses $H(F)^{\prime}$ and $H(K)$ hold and $M\|\eta\|_{1}<1$, then $(*)$ admits a solution.

Proof: As in the proof of Theorem 1, we can show that for every solution $x(\cdot) \in C(T, X)$ of $(*)$, we have $\|x\|_{C(T, X)} \leq M_{1}$. Then define $\widehat{F}(t, x)=F\left(t, p_{M_{1}}(x)\right)$. This has the same measurability and continuity properties as $F(t, x)$ satisfies $H(F)(4)$, (see the proof of Theorem 1) and $|\widehat{F}(t, x)| \leq \phi(t)$ a.e. with $\phi(\cdot) \in L_{+}^{1}$.

Let $\Gamma: C(T, X) \rightarrow P_{f}\left(L^{1}(X)\right)$ be defined by

$$
\Gamma(x)=S_{\widehat{F}(\cdot, x(\cdot))^{1}}
$$

Then from Papageorgiou [9] (see theorem 4.1) we get that $\Gamma(\cdot)$ is l.s.c. Apply theorem 3 of Bressan-Colombo [2] to get a continuous map $\gamma: C(T, X) \rightarrow L^{1}(X)$ such that $\gamma(x) \in \Gamma(x)$ for all $x \in C(T, X)$.

As in the proof of Theorem 1, let

$$
H=\left\{y \in C(T, X): y(t)=p(t)+\int_{0}^{t} K(t, s) g(s) d s, t \in T,\|g(t)\| \leq \phi(t) \text { a.e. }\right\} \text {. }
$$

This is bounded and equicontinuous. Let $R: H \rightarrow H$ be defined by

$$
R(x)(t)=p(t)+\int_{0}^{t} K(t, s) \gamma(x)(s) d s
$$

Since $\gamma(\cdot)$ is continuous, we can easily check that $R(\cdot)$ is continuous too. From the proof of Theorem 1, we know that it is $\hat{\alpha}$-condensing. So there exists $x=R(x)$. This is the desired solution of $(*)$. 
Acknowledgement: The author wishes to thank the referee for his/her corrections and constructive criticism.

\section{REFERENCES}

[1] Attouch, H., Convergence for Functions and Operators, Pitman, Boston 1984.

[2] Bressan, A., Colombo, G., Extensions and selections on maps with decomposable values, Studia Math. XC (1988), 69-85.

[3] Chuong, P.V., Existence of solutions for random multivalued Volterra integral equations, J. Integral Eqns. 7 (1984), 148-173.

[4] Diestel, J., Uhl, J.J., Vector Measures, Math. Surveys, Vol. 15, AMS, Providence, RI 1977.

[5] Klein, E., Thomson, A., Theory of Correspondences, Wiley, New York 1984.

[6] Lakshmikantham, V., Leela, S., Nonlinear Differential Equations in Abstract Spaces, Intern. Series in Nonlinear Math. Theory, Vol. 2, Pergamon Press, Oxford 1981.

[7] Lyapin, L, Hammerstein inclusions, Diff. Eqns. 2 (1976), 638-642.

[8] Papageorgiou, N.S., On the theory of Banach space valued multifunctions. Part 1: Integration and conditional expectation, J. Multivariate Anal. 17 (1985), 185-205.

[9] Papageorgiou, N.S., Convergence theorems for Banach space valued integrable multifunctions, Intern. J. Math. and Math. Sci. 10 (1987), 433-442.

[10] Papageorgiou, N.S., Existence and convergence results for integral inclusion in Banach spaces, J. Integral Eqns. and Appl. 1 (1988), 22-45.

[11] Ragimkhanov, R., The existence of solutions to an integral equation with multivalued right hand side, Siberian Math. Jour. 17 (1976), 533-536.

[12] Tarafdar, E., Vyborny, R., Fixed point theorems for condensing multivalued mappings on a locally convex topological space, Bull. Austr. Math. Soc. 12 (1975), 161-170.

[13] Wagner, D., Survey of measurable selection theorems, SIAM J. Control. Optim. 15 (1977), 859-903. 


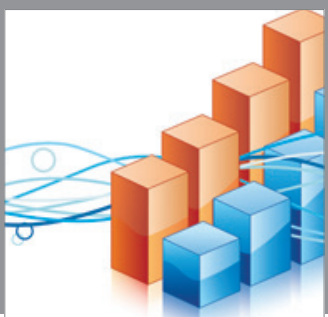

Advances in

Operations Research

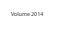

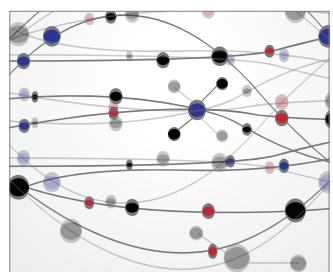

\section{The Scientific} World Journal
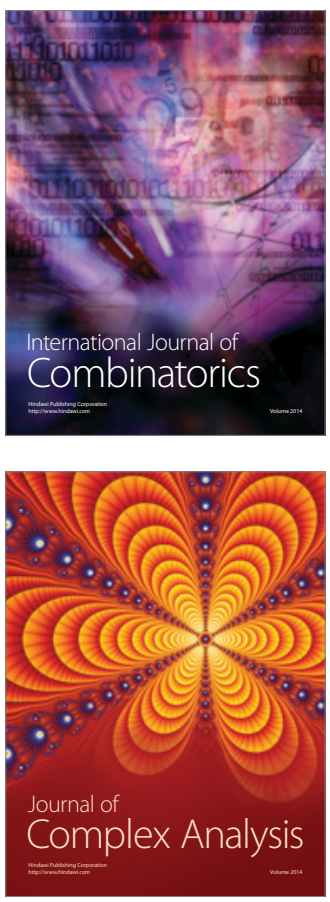

International Journal of

Mathematics and

Mathematical

Sciences
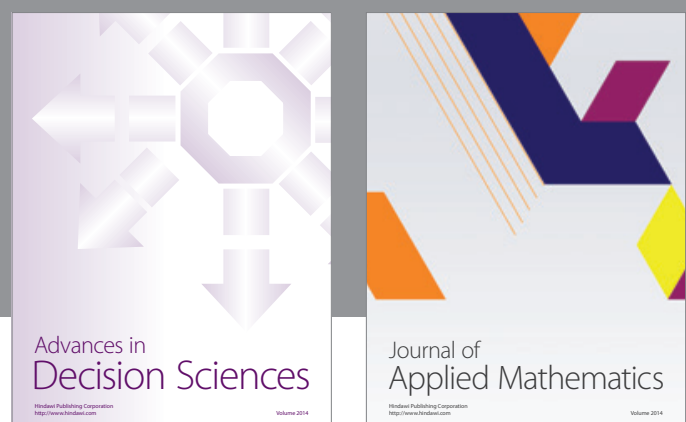

Journal of

Applied Mathematics
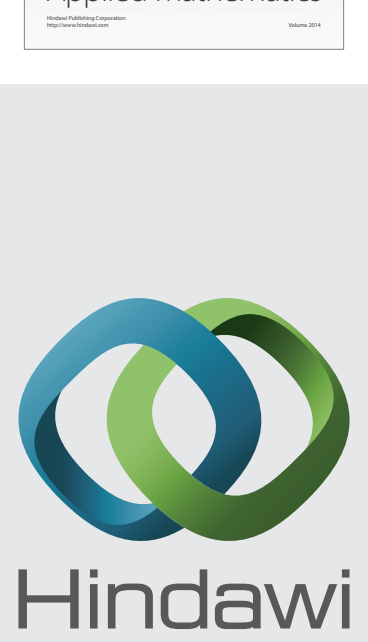

Submit your manuscripts at http://www.hindawi.com
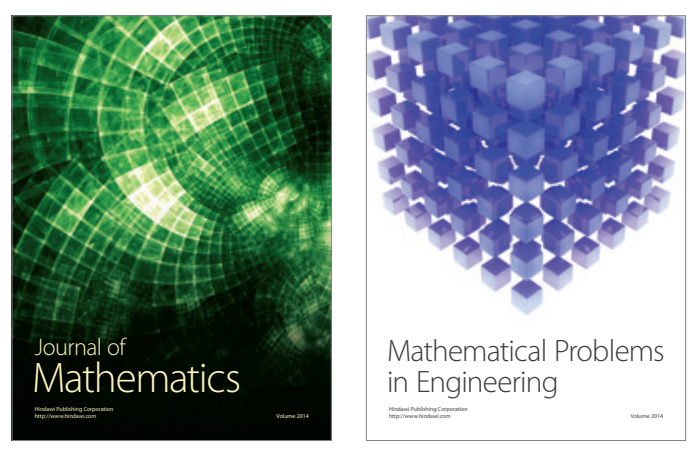

Mathematical Problems in Engineering
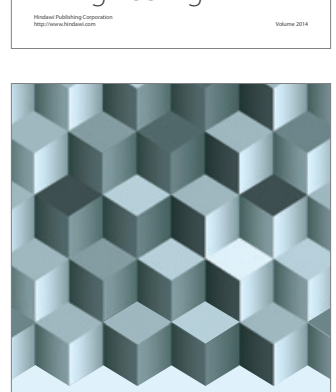

Journal of

Function Spaces
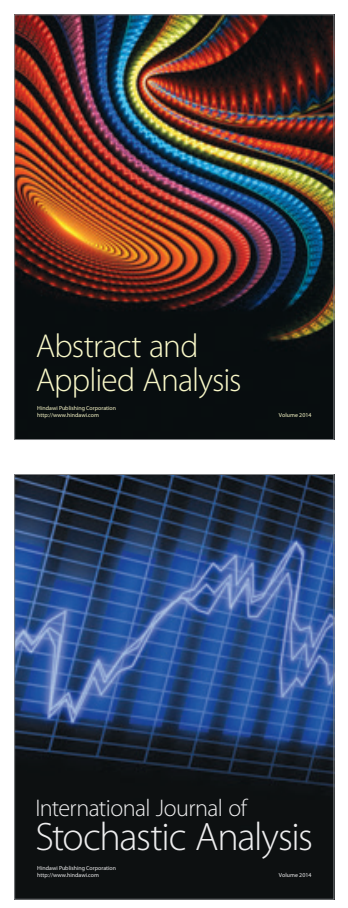

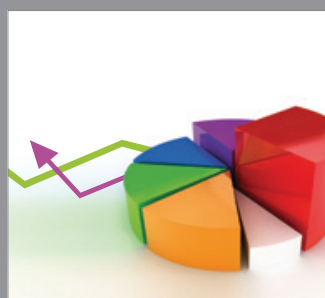

ournal of

Probability and Statistics

Promensencen
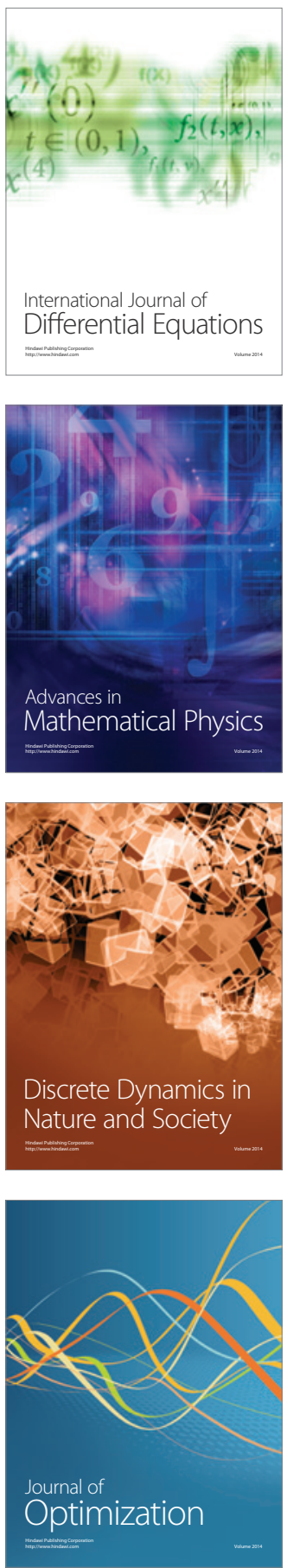